\title{
EBREI E MARRANI IN ITALIA IN UNA COMMEDIA RINASCIMENTALE
}

ROBERT C. MELZI

Widener University

La storia degli ebrei in Italia durante il Rinascimento è stata interpretata da molti storici in due maniere fondamentalmente differenti. Quelli che seguivano le teorie di Cecil Roth' e di Moses Sulvass ${ }^{2}$ vedevano nell'Italia rinascimentale come un Eden, in cui gli ebrei vivevano una vita quasi arcadica. Salo Baron ${ }^{3}$, d'altra parte, dava enfasi alle sevizie fatte subire agli ebrei da parte di una popolazione ostile, eccitata dalle feroci parole dei predicatori. Negli ultimi anni, studiosi americani ed europei hanno iniziato una revisione quasi completa delle terminologie sinora accettate ed hanno persino negato la validità di termini come "rinascimento" "ghetto" $e$ "barocco" quando applicati alla storia degli ebrei in Italia. Inoltre, il recente libro di Roberto Bonfil ${ }^{4}$ ci invita a rivedere senza esitazione tutte le teorie sinora accettate, mettendo in risalto l'importanza di fare ricerche su nuove fonti.

Gli studiosi in entrambi i campi si sono basati su cronache locali, su processi inquisitoriali, su storie, e su altri documenti che mettono in luce quali furono le traversie degli ebrei nella varie repubbliche, ducati, principati e regni dell'Italia d'allora, ma che non documentano la maniera in cui gli ebrei furono percepiti dalle masse che li circondavano e con le quali erano giornalmente in contatto. Lo stesso si dica dei documenti in ebraico che sono stati recentemente pubblicati da studiosi di storia economica e religiosa degli ebrei in Italia, documenti che rivelano come gli ebrei percepis-

\footnotetext{
${ }^{1}$ C. Roth, A History of the Marranos, Philadelphia 1932; IDEM, The History of the Jews of Italy, Philadelphia 1946.

${ }^{2}$ M. A. Shulvas, The Jews in the World of the Renaissance, Leiden 1973.

${ }^{3}$ S. W. Baron, A Social and Religious History of the Jews, New York 1952-1980.

${ }^{4}$ R. BonflL, Jewish Life in Renaissance Italy, Berkeley 1994.
} 
sero quelli che il Bonfil chiama "gli altri", ma non mettono in luce «la percezione degli ebrei da parte dell' "altro"

Il noto storico britannico, Peter Burke, suggerisce che, oltre che dei documenti sinora utilizzati, gli studiosi si servano di drammi rinascimentali come istrumenti storici; il Burke, però, avanza un'opinione che io non condivido, che cioè la letteratura non possa mai riflettere direttamente la realtà sociale e che i caratteri delle commedie siano sempre stereotipati '. La commedia rinascimentale in Italia fu, al contrario della tragedia, estremamente realistica e le commedie rinascimentali riflettono spesso le condizioni sociali esistenti al tempo in cui furono scritte. E ben noto che la commedia erudita in Italia fu modellata esattamente sulla commedia latina e che, nelle parole di un commediografo cinquecentesco, il Bentivoglio, essa ebbe come scopo precipuo di "essere specchio di natural costumi, imitazione del viver nostro, imagine del vero" ${ }^{6}$. Il Bentivoglio citava probabilmente Elio Donato che, a sua volta citava Cicerone con le parole "Comediam esse Cicero ait imitationem vitae, speculum consuetudinis, imaginem veritatis» '. Le parole del Bentivoglio sono quelle usate dal Varchi ${ }^{8}$, dal Gelli ${ }^{9}$ e da molti altri commediografi rinascimentali i quali non solo espressero teoricamente il precetto del realismo ma lo misero in atto, quando possibile, nelle loro commedie. Così, per esempio, Anton Francesco Grazzini, detto il Lasca, che fa dire al prologo nella Strega: «Primieramente in scena si conosce benissimo esser Firenze. Non vedi tu la cupola, bue?» per illuminare i suoi spettatori sull'ambiente della commedia ${ }^{10}$. In un'altra commedia il realismo è messo in luce da uno dei personaggi che si lamenta di non aver potuto dormire a causa del rumore fatto di buon mattino dai carri barcollanti sui ciottoli fiorentini, e ricorda le strade piene di fango lasciato dall'Arno in piena ". Nella Flora dell'Alamanni c'è una lista dei libri che il Marullo aveva portato

${ }^{5}$ P. Burke, The Historical Anthropology of Early Modern Italy, Cambridge 1987, pag. 23.

${ }^{6}$ E. Bentivoglio, Il Geloso, Venezia 1544, Prologo. Vedasi l'edizione curata da A. Dradi Moraldi, Torino 1972.

7 R. ANDREws, Scripts and Scenarios, Cambridge 1993, pag. 251.

${ }^{8}$ B. VARCHI, La Suocera, Firenze 1569, Prologo.

9 G. B. Gelli, La Sporta, Firenze 1566, Prologo.

10 A. F. Grazzini, La Strega, Venezia 1582, Prologo.

$"$ N. N., Comedia intitolata SINE NOMINE, Firenze, [Jacopo] Giunti, 1574, Atto I, scena iii. 
seco da Costantinopoli, col dettaglio di quanto sarebbero costati al tempo dell'Alamanni '2; nella Gostanza di Girolamo Razzi, il prologo (scritto probabilmente da Leonardo Salviati) dichiara la trama esser stata presa da "un caso a quello non molto dissimile esser, non sono ancor passati quindici anni, accaduto assai vicino al loco che questi orti e queste case rappresentano" ${ }^{13}$.

Evidentemente quanto gli autori del Cinquecento ci hanno lasciato non è la vox populi ma quanto essi credevano essere la percezione dell'ebreo da parte del popolo. E ben noto che gli autori di commedie rinascimentali o erano nobili essi stessi, o avevano un debito di riconoscenza col signore o con uno dei membri della corte. Come Werner Gundersheimer ha fatto osservare, il teatro rinascimentale è essenzialente antidemocratico; cito: "Indeed what I wish to propose today is that some of the most significant developments in the early history of the theatre in Northern Italy are precisely anti-democractic in their symbolic language and their theological implications" ${ }^{14}$. E la Riposio gli fa eco, osservando che «... è la Corte che "fa vivere" il Bibbiena, l'Aretino, il Belo. Molto più che nelle commedie. È nei prologhi che si trova la risposta al mondo della Corte» ${ }^{15}$. Questi pochi esempi che possono essere moltiplicati a diecine mi fanno credere che le commedie rinascimentali siano fonti storiche attendibili per la ricerca della visione degli ebrei negli occhi del resto della popolazione.

Essendo stato nominato ricercatore al Centro per gli studi sulla riforma e sul rinascimento dell'Università di Toronto, nel Canadà, passai l'estate passata leggendo molte delle commedie rinascimentali italiane conservate alla Fisher Library di quell'università. Delle settantasette commedie esaminate, trentotto furono stampate a Venezia, ventidue a Firenze, quattro a Roma, tre a Milano, due a Siena, due a Treviso, ed una ciascuna a Macerata, Mantova, Verona, Padova, Pavia e Parigi. La preponderanza delle stamperie di Venezia e Firenze non sembra essere essenzialmente significativa, dato che

12 L. Alamanni, La Flora, Firenze 1601, Atto III, scena iii.

13 G. RAzzl, La Gostanza, Firenze 1565, folio Ai verso e recto.

14 W. I. Gundersheimer, "Popular Spectacle and the Theater in Renaissance Ferrara", in Il teatro italiano del Rinascimento, Milano 1980, pag. 26.

15 D. Riposio, Nuova comedia v'apresento. Il Prologo nella Commedia del Cinquecento, Torino 1989, pag. 13. 
rappresenta solamente l'importanza delle case di Giolito de'Ferrari a Venezia e dei Giunti a Firenze ed è consistente con le statistiche forniteci dal Quondam ${ }^{16}$. Il lettore non sarà neppure sorpreso nell' apprendere che molte delle commedie furono preparate o furono rappresentate in occasione del carnevale, coerentemente alle teorie del Toschi, che vede nel carnevale l'origine del teatro italiano ${ }^{17}$.

Delle settantasette commedie esaminate, quindici mettono in scena un ebreo, o come protagonista, o come carattere secondario, o fanno riferimento a ebrei, a volte in maniera positiva, altre in maniera offensiva; questa percentuale del $19.5 \% \mathrm{mi}$ sembra essere estremamente elevata ed è confermata dalle ricerche attualmente in corso sulle commedie rinascimentali conservate nella Shakespeare Folger Library a Washington, D.C. Delle commedie esaminate a Toronto, una contiene un ebreo come protagonista; una descrive le sofferenze di una famiglia di marrani nella loro fuga dalla Spagna; tre usano la parola "ebreo" semplicemente come insulto; quattro usano la parola "marrano" come simbolo di inganno e duplicità; due rappresentano ebrei come vittime di crudeli beffe; due descrivono il prestito fornito da banchieri ebrei come una funzione socialmente importante per la comunità; due giustificano la disonestà dei mercanti ebraici ponendo parte della responsabilità sulle spalle di mercanti cristiani che erano consoci dietro le quinte; ed una viene infine alla difesa degli ebrei che sono descritti come vittime di un sistema fiscale talmente oneroso da forzarli ad essere disonesti.

Scelsi a caso le commedie, basandomi sulla lista preparata da Beatrice Corrigan ${ }^{18}$, e dando la preferenza non agli autori più famosi ma a commedie scritte da commediografi meno conosciuti che erano però giunte alla seconda o alla terza edizione, o che, nella prefazione, indicavano essere state ben ricevute dal pubblico. Inoltre, la scelta fu fatta in base alla trama e la preferenza fu data a quelle in cui si presentavano dissidi sociali o economici, o nelle quali la presenza di prostitute, lenoni, servi furbi, o figli rimasti al verde indicava la probabilità di incontrare personaggi in cerca di denaro. Il risultato delle mie investigazioni, al momento attuale, indica che

16 A. QuONDAM, "La letteratura in tipografia», in AA.VV., Letteratura italiana, Torino 1983, vol. II, pagg. 581-587; 687.

17 P. ToschI, Le origini del teatro italiano, Torino 1955, pag. 727.

18 B. Corrigan, Catalogue of Italian Plays 1500-1700 in the Library of the University of Toronto, Toronto 1961. 
molto di raro gli ebrei sono i protagonisti di commedie rinascimentali e, quando lo sono, rimangono, coerentemente alle parole del Burke, vuote marionette e caratteri stereotipati. I personaggi vivi e veri sono i servi che sono spesso i portavoce delle teorie sociali dell'autore e che fanno da va e vieni fra i padroni, giovani o vecchi che essi siano, e gli altri personaggi, ivi compresi i feneratori ebrei. Dalle mie investigazioni risulta inoltre che l'astio verso gli ebrei sembra essere inversamente proporzionale alla classe sociale e alla cultura del personaggio. E quindi evidente che ragazzacci cenciosi, certamente eccitati dalle parole dei predicatori, odiavano gli ebrei e volevano coprirlo di sassate; mentre i giovani nobili che si servivano dei feneratori per sopperire alla penuria economica imposta dall'avarizia dei padri, risconoscevano la necessità economica di avere un banchiere ebraico in città e ne tessevano le lodi, confermando quindi le teorie esposte da molti degli storici moderni ${ }^{19}$.

Fra le commedie esaminate vorrei soffermarmi sulla Comedia Intitolata SINE NOMINE, stampata a Firenze dai Giunti nel 1574, nella quale sono descritte le vicissitudini di ebrei o marrani giunti a Firenze dalla Spagna. La commedia è fra le meno conosciute del repertorio rinascimentale e persino la data di pubblicazione è in dubbio. L'Allacci la descrive come "Commedia (in prosa) intitolata sine Nomine, - in Firenze, nella stamperia de'Giunti 1554 [sic] in 8, d'Incerto Autore. - Jacopo Giunti, nella Lettera Dedicatoria di questa Commedia dice, che non si sapeva chi l'avesse composta; si stima però da alcuni, che sia di qualche Fiorentino, per quanto si può riconoscere da $i$ modi di dire" ${ }^{20}$. La copia da me esaminata indica la commedia essere stata stampata nel 1574 , data confermata dal Giunti stesso che, nella sua dedica del 15 dicembre 1573 «al molto Mag. et virtuoso Messer Tiberio Novellucci", esprime la sua intenzione di «farla per questo Carnovale stampare». Le poche copie esistenti

${ }^{19}$ Vedansi in special modo B. PULLAN, The Jews of Europe and the Inquisition of Venice 1550-1670, Totowa, N.J., 1983, e J. I. IsRAEL, European Jewry in the Age of Mercantilism, 1550-1750, Oxford 1985.

${ }^{20}$ L. AllacCI, Drammaturgia, Venezia 1755 [ristampa Torino 1966], pag. 724. 
negli Stati Uniti ${ }^{21}$ menzionano solo l'edizione del 1574; lo stesso si dica della copia esistente al British Museum ${ }^{22}$ e della descrizione fatta dal Brunet ${ }^{23}$ che fa menzione solo di un'edizione del 1574, sicché vorrei dedurre che la data addotta dall'Allacci è un semplice "lapsus calami». E pure evidente che la presente commedia non ha nulla a che vedere con la Commedia senza titolo del Beolco.

Non solo l'identificazione della commedia è, come si vede, estremamente problematica, ma il fatto che già nel prologo l'autore presenti Alonso, e i suoi figli, Fernando e Alvaro, come marrani fuggenti l'Inquisizione, aumenta le difficoltà, a causa del molteplice significato di questa parola, in Italia e altrove. Il problema è stato ben messo a fuoco da Uri Zolter che, non molti anni fa, scrivendo un saggio su Colombo, gli ebrei, i marrani ed i cattolici, ha imposto al suo lavoro il titolo "Columbus, Catholics, Marranos, and Jews. Who Was Who and What Was What?" che si potrebbe tradurre approssimatamente "Colombo, i cattolici, i marrani e gli ebrei. Chi erano e cosa voleva realmente dire il loro nome?» ${ }^{24}$, articolo in cui si trova un interessante riassunto delle teorie sinora avanzate sui marrani.

Senza entrare nella disputa sulla differenza fra marrani e nuovi cristiani, la parola "marrano" ha vari significati in varie lingue, in vari tempi. Si potrebbe ricercarne il significato in enciclopedie e dizionari, tenendo conto che, per quanto il lessicografo debba essere teoricamente al di sopra della mischia, egli è forzosamente lo specchio della società, e ne rivela quindi le forze, le debolezze, le idiosincrasie e i pregiudizi. Tale ricerca, però, ci porterebbe fuori dal soggetto della mia ricerca. Basti quindi segnalare che mentre in Ispagna la parola marrano venne a avere il significato di "persona scomunicata e maledetta", essa divenne in Italia il simbolo della duplicità. Il Corominas, forse, coglie nel giusto: «La idea de separar las dos acs. había nacido de la infortunada ocurrencia de Mariana

${ }^{21}$ Vedansi il National Union Catalogue Pre-1956 Imprints, London 1970, Vol. CXVII, pag. 217; e il Short-Title Catalog of Books Printed in Italy and of Books in Italian Printed Abroad 1501-1600 held in Selected North-American Libraries, Boston 1970, Vol I, pag. 447.

${ }^{22}$ BrITISH MUSEUM, Catalogue of Italian Books, 1465-1600, Londra 1958, voce "Giunti, House of".

${ }^{23}$ F. Brunet, Manuel du libraire, Parigi 1922, Vol. II, pag. 178.

${ }^{24}$ U. Z. Zolter, “Columbus, Catholics, Marranos, and Jews. Who Was Who and What Was What?» Lexington Theological Quarterly XXVIII (1993) 177-200. 
de traducir arbitrariamente por "sea anatema, marrano y descomulgado" la formula latina anathema maranatha sit, donde el aramaico maranatha "maldición" nada tiene en común, por el origen ni siquiera por el sentido, con marrano, improperio vulgar lanzado, no contra los herejes o descomulgados, sino contra los conversos sospechosos de ser relapsos; ... tanto éxito tuvo la idea que todavía la ampara la Acad. con su autoridad, atribuyendo a marrano el sentido de "persona maldita y descomulgada" que nunca tuvo precisamente" ${ }^{25}$. Corominas conclude che era impossibile aspettarsi la verità a causa de «... la enorme inverosimilitud de suponer una denominación fría y objetiva en el ambiente de odio y pasión brutal en que nació y vivió siempre este famoso vituperio" ${ }^{26}$. Notisi che in Italia l'edizione del 1612 del Vocabolario della Crusca ${ }^{27}$ non fa menzione di questa voce. Edizioni successive del Vocabolario si basarono sull'esempio di poeti e scrittori del Cinquecento e diedero alla parola il significato di "infedele", "misleale". Vedasi, per esempio l'edizione del 1733 che cita il Berni e il Guicciardini per giustificare il suddetto significato ${ }^{28}$. Finalmente, altre edizioni del Vocabolario aggiunsero, oltre al significato ereditato dagli spagnoli, la glossa di "sleale», "traditore»; "persona rozza e ignorante e un po' prepotente", e persino di "micidiale, pestifero, maligno, ecc.» ${ }^{29}$. Questa interpretazione è confermata dal Lessico Universale Italiano ${ }^{30}$ in cui si pone enfasi sulla duplicità e sulla mancanza di fede alla parola data, di cui furono tacciati i marrani ${ }^{31}$. A quanto pare, la taccia di duplicità era venuta ai marrani quando alcuni di essi furono espulsi da Venezia per esser stati coinvolti in una frode concernente l'importazione di grano alla Serenissima ${ }^{32}$. Notisi che in Italia la parola s'incontra pure con la grafia "martani», nome che ho spesso trovato

${ }^{25}$ J. Corominas - J. PASCual, Diccionario crítico etimológico de la lengua castellana, Madrid 1954, Vol. III, pag. 272.

26 Ibid.

27 Accademici della Crusca, Vocabolario degli Accademici della Crusca, Venezia 1612.

28 Accademici della Crusca, Vocabolario degli Accademici della Crusca, Firenze 1733 , voce "marrano".

${ }^{29}$ Accademici della Crusca, Vocabolario degli Accademici della Crusca, Firenze 1905, voce "marrano".

${ }^{30}$ Lessico universale italiano, Roma 1974, Vol. XIII, pag. 102.

${ }^{31}$ A. FARINelli, Marrano (Storia di un vituperio), Ginevra 1925, pag. 43-47.

32 B. Pullá, op. cit., pag. 170. 
nelle commedie consultate ${ }^{33}$. In Italia la parola è inoltre diventata semplice epiteto offensivo, come, per esempio, nella commedia $L e$ due cortigiane di Lodovico Domenichi, in cui il servo Vespa lancia l'insulto: "Va in hora mala tu e quanti marrani vennero mai di Spagna" ${ }^{34}$.

In questa più che in altre commedie l'autore sembra aver cercato di convincere il suo pubblico del realismo degli episodi descritti. Per esempio, una delle protagoniste, Teressa, spiega che «un fango sino al ginocchio", che ha invaso la strada presso la sua casa è stato causato dalla piena dell'Arno, prodotta a sua volta dal letto del fiume che da qualche anno in qua si è alzato. In maniera tipicamente fiorentina, che si potrebbe applicare al secolo ventesimo, la buona donna accusa il governo cittadino di non aver riparato la fogna come avrebbe dovuto fare ${ }^{35}$. D'altra parte, il padre, Alonso, si lamenta, nello stesso atto, di non aver amici e di trovarsi solo in paese straniero. Egli ha perso "l'amata patria» e si trova qui (a Firenze) "con poche pratiche e conoscenze»; le sue parole suonano prive di sentimento e piene di retorica dato che egli è, come si è già detto, null'altro che un fantoccio ed il suo soliloquio è modellato sulla miriade di lamenti incontrati in altre commedie rinascimentali. Alonso invoca la "dolce patria mia" e si chiede quando finiranno i suoi "tanti mali". Egli ha perso tutta la sua famiglia ed, essendo fuggito da Barcellona per vivere a Maiorca con solo un figlio, ne è stato cacciato per "fuggire il pericolo e la rabbia della severa Inquisizione" ${ }^{36}$, sicché ha persino dovuto mutare nome da Alonso a Roderigo. Vivendo solo a Firenze con il figlio, Fernando, Alonso ha incontrato una vedova che vorrebbe sposare, se non fosse per il timore di imporre una matrigna al suo diletto figlio. Nel frattempo, a sua insaputa, il servo, Padiglia, ha salvato l'altro suo figlio, Alvaro, che vive pure a Firenze ed è innamorato di una schiava, che, come vedremo più oltre, è stata comprata da suo padre.

La commedia sembra riflettere la situazione degli ebrei o dei marrani a Firenze intorno al 1573 e bisogna quindi chiedersi perché

\footnotetext{
${ }^{33}$ Vedasi, per es., S. Batt Aglia, Grande Dizionario della Lingua Italiana, Torino 1975, Vol. IX, pag. 835.

${ }^{34}$ L. Domenichi, Le Due cortigiane, Firenze 1563, Atto IV, Scena viii.

${ }^{35}$ Comedia SINE NOMINE, Atto I, Scena iii.

${ }^{36}$ Atto I, Scena i.
} 
l'autore descriva una famiglia di marrani che arriva direttamente da Barcellona, via Maiorca, mentre pare che nella seconda metà del secolo un buon numero di conversos arrivasse in Italia dopo aver passato un certo periodo di tempo nell'Impero Ottomano ${ }^{37}$. Comunque, dato il buon trattamento offerto ai conversos dal Granduca di Toscana, la scelta di Firenze sembra essere piuttosto logica. L'autore che è, come sembra, cristiano, doveva avere idee piuttosto confuse su chi fossero i marrani e da dove venissero; egli è però abbastanza al corrente delle sevizie subite dagli ebrei in Spagna, da descrivere Alonso e la sua immediata famiglia come gli unici scampati all'incendio, sia quello della loro casa, sia il fuoco al quale l'Inquisizione spagnola ha condannato gli altri suoi parenti.

Bisogna chiedersi non solo perché il nostro autore faccia arrivare il suo protagonista a Firenze da Barcellona, via Maiorca, e gli faccia pure fare una tappa a Padova e a Venezia. Per quanto sia estremamente difficile stabilire con precisione quale sia stata la rotta dei marrani nel loro viaggio dalla Spagna e il Portogallo, all'Italia, l'ignoto autore della nostra commedia sembra sapere che a Maiorca vi era una fiorente colonia de marrani ${ }^{38}$. Pare che egli non sia stato al corrente delle atrocità commesse su di essi dai maiorchini stessi ${ }^{39}$, dato che, sia il padre, sia il servo del figlio, descrivono solo le atrocità commesse dall'Inquisizione spagnola. Per quanto riguarda la tappa fatta a Venezia/Padova, gli storici sono più o meno concordi nell'ammettere che nel 1569 Venezia aveva dato il permesso agli ebrei levantini (che cioé, pur parlando spagnolo, si dichiaravano sudditi del Sultano) e a quelli ponentini, (eufemismo per marrani, cioé ebrei spagnoli e portoghesi) di ritornare alla Serenissima ${ }^{40}$. Inoltre, l'ordine di espulsione del 1571 non fu fatto osservare assolutamente e quanti marrani avevano lasciato Venezia, vi ritornarono fra il 1573 e il $1574{ }^{41}$. Una tappa nel territorio della Serenissima sarebbe quindi storicamente plausibile.

Il nostro autore usa la parola «marrani» col significato di «emi-

37 J. I. ISRAEL, op. cit., pag. 45.

38 J. I. IsRael, op. cit., pag. 35 e M. Forteza PINA, Els Descendents dels conversos de Mallorca. Quatre mots de la veritat, Mallorca 1966, recensito da J. M. Millás Vallicrosa in Sefarad XXVII (1967) 152-153.

${ }^{39}$ C. Roth, A History of th Marranos, pag. 93.

${ }^{40}$ B. Pullan, op. cit., pag. 150.

${ }^{41}$ J. I. ISRAEL, op. cit., pag. 47. 
grati arrivati recentemente dalla Spagna e di molto probabile origine ebraica». Questa è, approssimativamente, la stessa interpretazione data alla parola da molti storici moderni; l'Israel, per esempio, definisce il marrano come "an ostensible Christian who is a CriptoJew» ${ }^{42}$. Secondo il Pullan, i marrani erano «immigrants from Portugal and from Spain who had Jewish blood and some experience in living, at least outwardly, as Catholics» ${ }^{43}$. Il Pullan fa inoltre una distinzione fra tre categorie di marrani, cioé cattolici senza fede che erano, allo stesso tempo, ebrei senza conoscenza della loro religione; eccellenti cattolici, che sarebbero rimasti cristiani se fossero stati lasciati in pace; e finalmente persone religiosamente indifferenti ad entrambe le fedi. Tale distinzione, che deriva da quella fatta da Julio Caro Baroja, è discutibile, come sono sospette molte delle teorie avanzate dal Caro Baroja ${ }^{44}$. E difficile stabilire con esattezza a quale categoria di marrani (se queste categorie in realtà esistessero) il nostro autore ponga Alonso. Di sua moglie, si sa che pratica il cattolicesimo, dato che va a messa, con quanta sincerità non sappiamo. Non è nemmeno chiaro se gli eroi della nostra commedia siano a contatto con la comunità ebraica di Firenze che sappiamo essere fiorente a quell'epoca, per quanto confinata nel ghetto ${ }^{45}$.

I marrani sono, quindi, in questa commedia null'altro che un pretesto per introdurre l'ormai frusta agnizione nel contesto di rifugiati ebrei dalla Spagna i cui figli avrebbero potuto essere stati verosimilmente separati in gioventù ed esser quindi risconociuti nel quinto atto della commedia a lieto fine. L'altro figlio, Alvaro, ha un servo, Padiglia, che sa che il suo padrone è di origine ebraica, e gli ricorda sovente il fuoco della vecchia casa a Barcellona ${ }^{46}$; contrariamente ai desideri della chiesa che voleva una barriera assoluta fra ebrei e cattolici, Padiglia dichiara amore filiale per il suo padrone, marrano che egli sia, amore ricambiato da Alvaro, che ha perso madre e fratelli e crede aver perso pure il padre. Apparentemente il Giunti, nel 1574, non aveva ancora bisogna di «licenza de' superiori»

42 J. I. IsRael, op. cit., pag. 3.

43 B. Pullan, op. cit., pag. xiii.

44 J. CARo BARoja, Los judios en la España moderna y contemporánea, Madrid 19782 . Vedasi la severa recensione fatta alla prima edizione da J. L. Lacave in Sefarad XXIII (1963) 372-388.

45 J. I. ISRAEL, op. cit., pag. 22.

46 Comedia SINE NOMINE, Atto I, Scena ii. 
per stampare la sua commedia e poteva quindi mettere in scena un marrano servito da un servo cristiano. Non si sa, evidentemente, quale fosse la politica ufficiale a Firenze, a questo momento, verso marrani che, almeno ufficialmente, si facevano passare per cristiani.

La moglie di Alonso, che ignora la presenza del perduto marito a Firenze, è pure una delle fredde figure della commedia erudita, posta lì ad attendere l'arrivo di Alonso, il riconoscimento e il dénouement. I servi, invece, sono pieni di invenzioni e di entusiasmo. Menia, la serva di Alonso, vorrebbe che il padrone entrasse a casa della vedova vestito di un camiciotto azzurro, uno di quelli usati dai ragazzi che vendono il lino. Tingo, il velettaio, propone al giovane Fernando di cambiare vesti con lui, per entrare a casa della ragazza con la scusa di vendere "uno sfilato". E Menia vorrebbe che l'altro figlio, Alvaro, vestito da servetta, entrasse nella casa di Teressa dove Quirilla vive, per incontrarsi con la giovane.

Sfortunatamente, l'anonimo autore è commediografo di second' ordine e la commedia degli errori che potrebbe essere realmente divertente e piena di sorprese, diviene null'altro che una noiosa enumerazione delle passate traversie ed una serie di calchi su altre commedie rinascimentali. Rimane solo il salace episodio di Alvaro che, vestito da donna, deve essere lavato dalla nutrice, con i risultati che ognuno può immaginare. La faccenda si ingarbuglia quando il padre, Alonso, per rimaner solo nella sua avventura amorosa, vuole inviare suo figlio, Fernando, a Pistoia a incassare cento ducati che l'Abate gli deve. Fernando, però, ha un appuntamento con Aldanza, e se non vi andasse, perderebbe il suo amore. L'unica soluzione è di prendere a prestito i cento scudi, darli al padre, andare all'appuntamento amoroso e, il giorno dopo, prendere il cavallo, andare a Pistoia, incassare il denaro dall'Abate, e ritornare i cento scudi a Isacco. Tingo, il velettaio, si è occupato del prestito; è andato da Isac, che egli conosce bene e che lo serve immediatamente. L'amicizia tra Tingo ed Isacco è di vecchia data; Tingo dice che "non prima Isac m'ebbe visto che mi spacciò, perché è mio amico vecchio". Tingo fà inoltre alcune osservazioni estremamente interessanti sul valore sociale d'aver un banchiere ebreo in città. Rivolgendosi a Fernando, Tingo così esprime le sue opinioni: «Et a dire il vero l'hebreo per questi simili casi repentini da giovani, le voglie de quali non aspetton tempo, \& dica chi vuole, è una cosa santa in una 
citta» ${ }^{47}$. Il "dica chi vuole", suona stranamente nuovo, se Tingo, come pare, ha in mente le autorità ecclesiastiche che, per evitare il contatto fra ebrei e cristiani, alcuni anni prima avevano creato un ghetto. Se i feneratori ebrei siano stati espulsi da Firenze prima del 1573 non è chiaro, ma il nostro autore, a quanto pare, non ne è al corrente. Egli sembra, però, avere una fede quasi sconfinata nei banchieri ebrei; lo stesso Tingo, a Fernando che gli chiede se si sia fatto dare una ricevuta per gli argenti che ha impegnato con Isacco, gli risponde di non averlo fatto perché «l'hebreo non t'ingannerebbe». Queste parole, dette da Tingo, sembrano esprimere l'opinione dell'anonimo autore che non è certamente antisemita. Se l'autore della commedia sia stato un ebreo lui stesso e si sia nascosto sotto l'usbergo dell'anonimità è una possibilità molto remota, dato che un ebreo sarebbe stato molto più al corrente della vera situazione dei suoi correligionari e di quella dei marrani.

Presa isolatamente, la Comedia intitolata SINE NOMINE non rende molti indizi e non svela nessun segreto; se da essa si possa inferire che la vita dei marrani, finchè rimanevano formalmente ligi al cristianesimo e non davano espressioni esteriori di cripto-semitismo, fosse abbastanza pacifica è una teoria interessante che però la commedia non conferma in nessuna maniera. Presa però nel contesto delle altre commedie da me esaminate, credo che questa commedia confermi i miei sospetti che gli ebrei, anche a Firenze, durante un periodo di notoria pressione religiosa, potessero vivere un po' meglio di quanto altri documenti hanno rivelato sinora. Spero che le mie ricerche in altre commedie, in altre biblioteche, potranno confermare questa mia prima impressione.

${ }^{47}$ Comedia SINE NOMINE, Atto. I, Scena iii. 


\section{RESUMEN}

Por su extremado realismo, las comedias del Renacimiento italiano pueden utilizarse como fuentes históricas para evaluar las costumbres sociales, comerciales y religiosas de la época. La Comedia SINE NOMINE, escrita por un autor desconocido e impresa en Florencia por los Giunti en 1574, relata el traslado de una familia marrana desde Barcelona a Florencia. Pese a caer a menudo en las redes de una "commedia erudita" italiana, la obra es de gran interés porque ayuda a una mejor comprensión de las condiciones en las que vivían los judíos y los marranos en la Florencia del Renacimiento.

\section{SUMMARY}

Because of their extreme realism, Italian Renaissance comedies can be used as historical tools, in order to gauge social, commercial, and religious customs in Sixteenth Century Italy. The Comedia SINE NOMINE, written by an unknown author, printed in Florence by the Giuntis in 1574, relates the flight of a marrano family from Barcelona to Florence. In spite of being often caught in the web of the Italian "commedia erudita", the play is of great interest because it helps us to better understand the conditions under which Jews and marranos lived in Renaissance Florence. 